\title{
Enhancing solar cells with photochemical upconversion
}

Tim Schulze, Klaus Lips, and Timothy W. Schmidt

Triplet-triplet annihilation in organic molecules increases the photocurrent of thin-film solar cells.

Photovoltaics have the potential to become a major contributor to future sustainable energy generation. To this end, device efficiency needs to be brought beyond present physical limits. Solar cells cannot absorb light with less energy than the bandgap (absorption threshold of the photovoltaic absorber material), and consequently this energy source is usually uncaptured. Upconversion (UC) devices harvest those unused subthreshold photons behind the solar cell, create one higher-energy photon out of (at least) two transmitted photons, and radiate upconverted light back toward the solar cell, thus expanding the usable solar spectrum (see Figure 1). Key requirements for UC units are broad absorption and high UC quantum yield under even the low-intensity incoherent illumination that is available to solar energy conversion devices.

Upconversion was proposed as a means to increase solar cell efficiency about a decade ago, ${ }^{1}$ and research has since then been primarily focused on UC processes occurring in lanthanide-doped glasses or nanoparticles. ${ }^{2}$ Although transitions between the rare earth $4 f$ orbitals lie in an energy range promising for combination with common crystalline silicon solar cells, UC systems based on erbium (Er) or ytterbium (Yb) suffer from weak absorption by Laporte-forbidden transitions, a narrow absorption bandwidth, and the resulting low efficiency. Recently, triplet-triplet annihilation (TTA) in organic molecules has been demonstrated to be an alternative UC mechanism highly promising for solar energy application. ${ }^{3}$ It proceeds efficiently even for low photon flux and displays highly tunable absorption characteristics.

The key to high quantum yield from TTA-UC lies in the combination of two different classes of organic molecules dissolved together in organic solvents or in a polymeric host matrix. ${ }^{4} \mathrm{~A}$ socalled sensitizer species, which in many cases is a metalated porphyrin, absorbs a low-energy photon (process 1 in Figure 2) and undergoes fast intersystem crossing (ISC, process 2), whereby

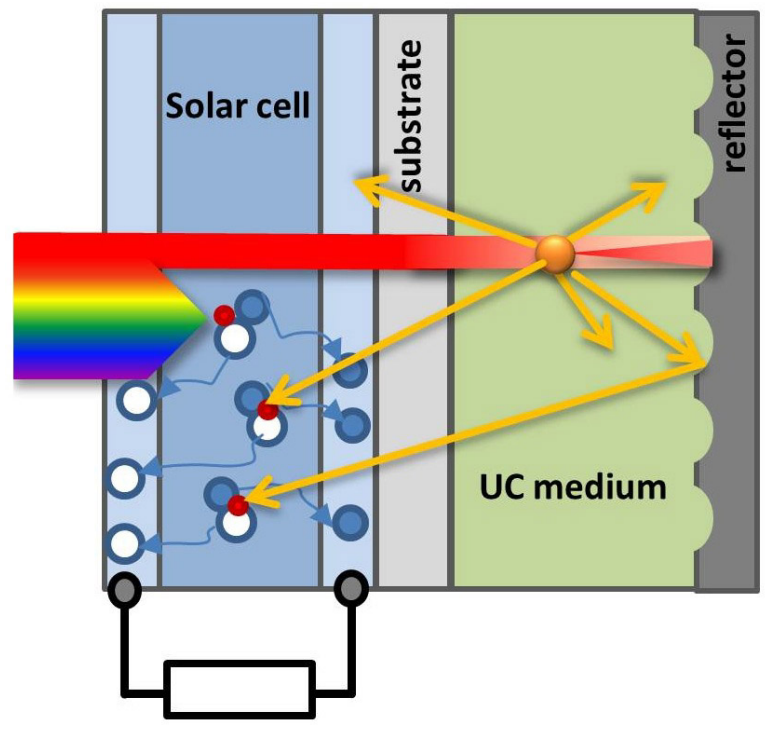

Figure 1. Principle of a solar cell assisted by upconversion (UC). The creation of usable electron-hole pairs in the photovoltaic absorber material (left) is augmented by light, usually transmitted unused, which is converted to higher photon energies in the UC unit (right).

the spin of the exciton changes from 0 (singlet, electron and hole spin cancel) to 1 (triplet, electron and hole spin add). This change of spin state is facilitated by the heavy metal in the porphyrin center. The resulting triplet state cannot radiatively relax back to the ground state and is therefore long-lived. Interaction with a second class of molecule transfers the triplet energy to the socalled emitter species by triplet energy transfer (TET, process 3 ). Successive interaction of two emitters yields TTA (process 4), which combines the two triplet excitons to create one higherenergy singlet state. The singlet state quickly relaxes back to the ground state under efficient emission of a high-energy photon (process 5).

We recently explored the feasibility of TTA-UC for expanding the spectral harvest of thin-film solar cells. We combined

Continued on next page 
TTA-UC systems comprising tailored molecular sensitizer species ${ }^{5}$ with amorphous silicon (a-Si) solar cells, ${ }^{6,7}$ with organic bulk heterojunction solar cells, ${ }^{8}$ and with dye-sensitized solar cells. ${ }^{9}$ We defined a figure of merit (FOM) for UC-assisted solar cells, which takes into account the inherent nonlinearity of the UC process in the benchmarking of different UC systems. Usually, UC systems are measured under highly concentrated illumination, which hinders a fair comparison of UC units measured under different conditions. Our FOM traces the UC performance back to conditions equivalent to the standard operation of a solar cell, ${ }^{6}$ and equals the photocurrent increase by UC under 1-sun conditions.

Using the FOM for guidance, we concentrated on increasing the performance of the solar cell/UC assembly. Shifting the sensitizer absorption into the IR by extending the $\pi$-system of the palladated sensitizer porphyrin led to a fourfold increase of the $\mathrm{FOM}^{6}$ compared with the original sensitizer species, using 130nm-thick hydrogenated a-Si (a-Si:H) cells. This preliminary result already beats the state of the art for a-Si:H cells augmented by rare-earth UC by a factor of about $200 .{ }^{10}$ Combining the UC unit with a back-reflecting surface helps to multipass the incident light as well as to out-couple the upconverted light, and led to a consecutive increase of the $\mathrm{FOM}^{7}$ by another factor of 3 to about $2-3 \times 10^{-4} \mathrm{~mA} / \mathrm{cm}^{2}$. Roughly the same level of photocurrent enhancement could be obtained for organic bulk
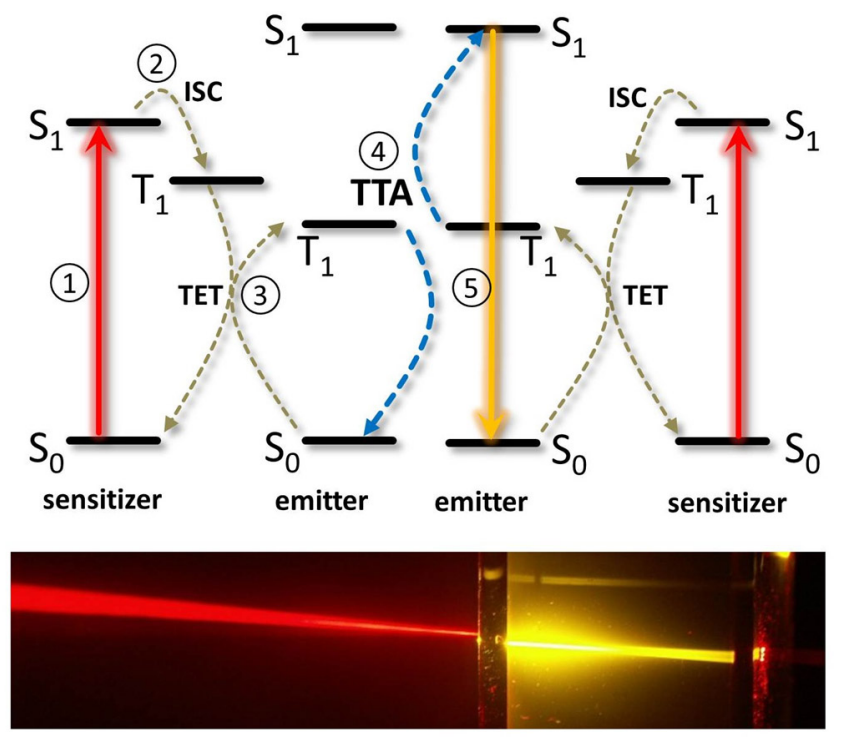

Figure 2. Top: Working principle of triplet-triplet annihilation upconversion (TTA-UC) shown on a schematic energy diagram. A key role is played by the long-lived triplet states $T_{x}$ of the organic molecules, which cannot decay quickly due to quantum-mechanical laws. Bottom: Picture of the TTA-UC effect. ISC: Intersystem crossing. $S_{x}$ : Singlet states. TET: Triplet energy transfer. heterojunction solar cells ${ }^{8}$ as well as for dye-sensitized solar cells with a fully device-integrated UC unit. ${ }^{9}$ The latter result was measured under illumination conditions very similar to device operation, reaching concentration factors as low as 3 suns. Our current record FOM stands at $7.6 \times 10^{-4} \mathrm{~mA} / \mathrm{cm}^{2}$, obtained with an a-Si:H solar cell optimized for low parasitic absorption. ${ }^{8}$

In summary, we have shown cumulative FOM improvements of roughly three orders of magnitude compared with lanthanide-based UC and a first move toward device-integrated UC units. However, the photocurrent increase under 1 sun achieved to date is still far from commercially relevant. We are therefore now working to increase the UC yield under lowillumination conditions. We are following two strategies in parallel and have recently obtained initial, promising results. First, we are increasing the dye concentration by tethering the UCactive molecules onto nanosized support structures to increase the steady-state triplet density and therefore the UC yield. ${ }^{11}$ Second, we are focusing the incident light locally in the UC layer using micro-optical structures such as parabolic back-reflectors. ${ }^{12}$ In combination, these strategies will enable us to exploit nearfield optical effects such as plasmonic resonances or wave-guide modes once characteristic absorption lengths in the 10-100nm range have been reached, which should make further increases in the UC yield possible. We are confident that TTA-UC will contribute significant photocurrent to high-gap solar cells and photoelectrochemical devices for fuel production in the near future.

T. F. S. acknowledges a Feodor-Lynen fellowship from the Alexander von Humboldt Foundation. T. W. S. acknowledges Australian Research Council funding FT130100177, DP110103300, and project funding from the Australian Renewable Energy Agency.

\section{Author Information}

Tim Schulze and Klaus Lips

Helmholtz-Zentrum Berlin für Materialien und Energie GmbH Berlin, Germany

\section{Timothy W. Schmidt}

The University of New South Wales

Sydney, Australia

\section{References}

1. T. Trupke, M. A. Green, and P. Würfel, Improving solar cell efficiencies by upconversion of sub-band-gap light, J. Appl. Phys. 92, pp. 4117-4122, 2002.

2. M. Haase and H. Schäfer, Upconverting nanoparticles, Angew. Chem. Int'1 Ed. 50 (26), pp. 5808-5829, 2011. 
3. S. Baluschev, T. Miteva, V. Yakutkin, G. Nelles, A. Yasuda, and G. Wegner, Up-conversion fluorescence: noncoherent excitation by sunlight, Phys. Rev. Lett. 97, p. $143903,2006$.

4. Y. C. Simon and C. Weder, Low-power photon upconversion through triplet-triplet annihilation in polymers, J. Mater. Chem. 22, p. 20817, 2012.

5. T. Khoury and M. J. Crossley, A strategy for the stepwise ring annulation of all four pyrrolic rings of a porphyrin, Chem. Commun. 46, pp. 4851-4853, 2007.

6. Y. Y. Cheng, B. Fückel, R. W. MacQueen, T. Khoury, R. G. C. R. Clady, T. F. Schulze, N. J. Ekins-Daukes, et al., Improving the light-harvesting of amorphous silicon solar cells with photochemical upconversion, Energy Environ. Sci. 5, pp. 6953-6959, 2012.

7. T. F. Schulze, Y. Y. Cheng, B. Fückel, R. W. MacQueen, A. Danos, N. J. L. K. Davis, M. J. Y. Tayebjee, et al., Photochemical upconversion enhanced solar cells: effect of a back reflector, Aust. J. Chem. 65, pp. 480-485, 2012.

8. T. F. Schulze, J. Czolk, Y.-Y. Cheng, B. Fückel, R. W. MacQueen, T. Khoury, M. J. Crossley, et al., Efficiency enhancement of organic and thin-film silicon solar cells with photochemical upconversion, J. Phys. Chem. C 116 (43), pp. 22794-22801, 2012.

9. A. Nattestad, Y. Y. Cheng, R. W. MacQueen, T. F. Schulze, F. W. Thompson, A. J. Mozer, B. Fückel, et al., Dye-sensitized solar cell with integrated triplet-triplet annihilation upconversion system, J. Phys. Chem. Lett. 4, pp. 2073-2078, 2013.

10. J. de Wild, J. K. Rath, A. Meijerink, W. G. J. H. M. van Sark, and R. E. I. Schropp, Enhanced near-infrared response of a-Si:H solar cells with $\beta$-NaYF ${ }_{4} Y^{3+}(18 \%), \mathrm{Er}^{3+}$ (2\%) upconversion phosphors, Sol. Energy Mater. Sol. Cells 94, pp. 2395-2398, 2010. 11. R. W. MacQueen, T. F. Schulze, T. Khoury, Y. Y. Cheng, B. Stannowski, K. Lips, M. J. Crossley, and T. Schmidt, Nanostructured upconverters for improved solar cell performance, Proc. SPIE 8824, p. 882408, 2013. doi:10.1117/12.2026907

12. T. F. Schulze, Y. Y. Cheng, T. Khoury, M. J. Crossley, B. Stannowski, K. Lips, and T. W. Schmidt, Micro-optical design of photochemical upconverters for thin-film solar cells, J. Photon. Energy 3 (1), p. 034598, 2013. 